\title{
Validation of the Beck Depression Inventory II among the Sinhalese speaking population in Sri Lanka
}

\author{
A Rodrigo, KALA Kuruppuarachchi, A Pathmeswaran
}

\section{Background}

Depression is an important public health issue in Sri Lanka, yet it is often missed or misdiagnosed, hence the development of valid and reliable diagnostic instruments for depression is of importance.

\section{Aims}

To examine psychometric properties of the Sinhalese version of the Beck Depression Inventory II (BDI-II), and to determine the cut off score for the diagnosis of depression.

\section{Method}

The linguistic equivalence of the scale was verified by a back-translation method. The final translation was administered to 956 randomly selected adults from the Ragama and Ja-Ela $\mathrm{MOH}$ areas.

\section{Results}

The mean age of the study population was 31.3 years with a majority being female (56.2\%). Participants were invited to complete the Sinhalese version of the
BDI II. The participants were also independently assessed for depression. Of the participants, one hundred and eight (11.2\%) were depressed. The scale showed a high level of internal consistency (Cronbach's $\alpha$ 0.93). Exploratory factor analysis showed a twofactor structure (cognitive-affective and somatic), which was similar to the original model by Whisman et al. Criterion validity was satisfactory. Based on the receiver operating characteristics curve a cut-off score of 16 was determined to be the most appropriate cut off value for the detection of depression in this population group.

\section{Conclusion}

The Sinhalese version of the BDI-II is a psychometrically robust tool that can be used to assess for depression in Sri Lanka.

Key words: depression, screening, validation, Sri Lanka

SL J Psychiatry 2015; 6(2) 20-24

\section{Introduction}

\section{Depression}

Depressive disorder is a serious, recurrent mental illness associated with diminished role-functioning, quality of life and increased mortality. The Global Burden of Disease Study 2010 showed depression to be the second leading cause of disability, accounting for 63 million years lived with disability (YLDs) and 9.6\% of all YLDs (1). The World Mental Health Survey conducted by World Health Organization in 2011 reported average lifetime prevalence estimates of depressive disorders to be $14.6 \%$ and $11.1 \%$ in high income and low to middle-income countries respectively (2). A Sri Lankan epidemiological study conducted in Colombo in 2006/7 showed that the lifetime prevalence of depression was $11.2 \%$ - on par with global prevalence (3). This study further demonstrated significant functional impairment linked with depression and a clear association between poverty and depression (OR=1.76, 95\%CI 1.30-2.36). Perera et al found prevalence of depressive symptoms in $57.7 \%$ of adolescents in Sri Lanka, a considerably higher rate than rates reported in U.S. adolescents (15-45\%) (4). A study conducted in Northern Sri Lanka, where active combat took place, in the post-war period recorded significant depressive symptoms in $22.2 \%$ adults (5). These studies demonstrate that depression is a major health issue in Sri Lanka. Consequences of depression include poverty, suicide with Sri Lanka having one of the highest rates in the world - substance misuse and non-communicable medical problems $(3,5)$.

Despite the high prevalence, and significant associated impairment and economic burden, depression is undertreated in Sri Lanka (3-7). It has been hypothesized that failure to identify depression is a main contributing factor to this situation.

\section{Missed diagnosis and misdiagnosis of depression}

Primary care studies show that despite depression being a common problem it is substantially under-diagnosed and undertreated (8, 9). A meta-analysis involving 50,371 patients reported primary care physicians correctly identified depression in $47.3 \%$ of cases and recorded depression in their notes in $33.6 \%$ (8). This study also suggested that misdiagnosis of depression is more common than missed diagnosis.

Approximately two thirds of patients with depression in primary care present with somatic symptoms and these 
patients are often misdiagnosed (9). Sri Lankan patients with depression are known to present with somatic symptoms, and this too may contribute towards missed or misdiagnosis (10). Valid screening tools for the detection of depression would help the primary care physicians identify this disorder, and has been shown improve outcomes internationally $(11,12)$.

\section{Screening tools and rating scales}

Contemporary psychiatry emphasises use of objective measures not only for identifying disease but also for evaluating treatment outcome and severity of symptoms (13). However clinicians are already overburdened, and adding further evaluations with rating scales to this workload is unlikely to meet with success (14). As Zimmerman et al pointed out a clinically useful rating scale should be reliable, valid, brief, self administered, clinically relevant and useful (15).

The Beck Depression Inventory II (BDI II) is a commonly used self-rating screening tool and rating scale for depression (16). The BDI II has good psychometric properties, high internal consistency, good test-retest reliability, and good construct and concurrent validity and discriminant validity in other cultures (17). The BDIII had not been validated for the Sri Lankan population and the aim of this study was to validate this scale among a Sinhalese speaking population in Sri Lanka (18).

\section{Methodology}

To preserve equivalence in cross-cultural adaptation of the BDI-II, we followed the guidelines proposed by Guillemin et al. (1993), including back-translation techniques (19). The BDI-II was translated into Sinhalese by two Sri Lankan psychiatrists. The preliminary Sinhalese translation was then back-translated by independent bicultural experts, an anesthetist and a senior English language teacher, under the license of Pearson Inc (USA). The Structured Clinical Interview for DSM Disorders (SCID) and psychiatric evaluation were used as the 'gold standards' to determine the presence or absence of depression among participants.

The study was conducted among randomly selected adults residing in the Ragama and Ja-Ela MOH areas. Those who were not conversant in the Sinhala language and who were unable to complete the questionnaires by themselves were excluded from the study. Following the completion of the BDI-II, all participants were interviewed by a psychiatrist on the same day or following day, for the assessment of the presence or absence of depression. The psychiatrist was blind to the BDI scores of the individuals. Basic socio demographic characteristics were noted separately. The study was approved by the Ethical review committee of the Faculty of Medicine, University of Kelaniya. Informed written consent was obtained from all participants and permission was taken from Pearson Assessment Inc. USA, after making necessary payments, for the use of the BDI II.

\section{Results}

One thousand one hundred and sixty six people participated in the study, of which 956 participants completed the both the questionnaire and the interview. Participant ages ranged from 19 to 66 years (mean age 31.3 years). The study sample included more females (56.2\%) and there was no age difference by gender (mean age among females- 33.8 years, SD 8.6 years; mean age in males- 28.1 years SD 9.0 years).

The SCID and psychiatric evaluation demonstrated 108 (11.3\%) participants were depressed.

Almost half (45\%) of participants scored 1 or more in item 10 "crying" whereas $86 \%$ scored 0 for item 14 "worthlessness". In response to the item 'changes in sleeping pattern', 27.0\% reported getting less sleep and $15.8 \%$ reported that they were sleeping more. For 'changes in appetite', $22.7 \%$ reported decreased appetite and $6.6 \%$ indicated increased appetite.

The Cronbach's alpha coefficient for the Sinhalese BDIII was 0.93 and the mean inter-item correlations was 0.42 , indicating good internal consistency and item homogeneity. The correlations ranged from 0.20 (worthlessness) to 0.81 (crying). The means, standard deviations and corrected item-total correlations of the 21 items are presented in Table 1.

\section{Exploratory factor analysis}

The data met the Kaiser-Meyer-Olkin criteria for sample adequacy (MSAs 0.94), as well as those for Bartlett's test of sphericity, (d.f.s210, P-0.000). Screen plot identified two factors to be extracted from five factors that were obtained, based on the eigenvalue criteria. The first principal component accounted for $45.7 \%$ of the variance and the first two components accounted for $53.8 \%$ of the variance. After promax rotation, all items showed salient factor loadings for one of the two factors. Details are shown in Table 2.

\section{Criterion validity}

Although the BDI-II was not specifically designed for diagnostic purposes, it assesses the presence and severity of depressive symptoms, and patients with major depression should have higher BDI-II scores on average than patients without this diagnosis. Analyses indicated that patients with a diagnosis of current major depression had significantly greater BDI-II total scores (M 30.81, SD 8.69) compared with patients without this diagnosis (M-6.14, SD -6.01; df-120.4 $\mathrm{p}<0.001$ ). The mean total BDI II score was 7.84 .

\section{ROC curve and cut off score}

While Beck et al. suggested that a score of 14 or greater was indicative of at least mild depression, they also encouraged the selection of a cut off score based on the 
Table 1. Means, standard deviations and corrected item-total correlations of BDI II

\begin{tabular}{llll} 
Symptom & Mean & S.D. & $r$ tot \\
1. Sadness & 0.33 & 0.61 & 0.71 \\
2. Pessimism & 0.34 & 0.73 & 0.70 \\
3. Past failure & 0.28 & 0.63 & 0.62 \\
4. Loss of pleasure & 0.45 & 0.76 & 0.61 \\
5. Guilty feelings & 0.37 & 0.63 & 0.53 \\
6. Punishment feelings & 0.56 & 0.84 & 0.57 \\
7. Self-dislike & 0.27 & 0.54 & 0.62 \\
8. Self-criticalness & 0.62 & 0.95 & 0.60 \\
9. Suicidal thoughts/wishes & 0.24 & 0.54 & 0.66 \\
10. Crying & 0.81 & 0.99 & 0.45 \\
11. Agitation & 0.62 & 0.85 & 0.71 \\
12. Loss of interest & 0.37 & 0.71 & 0.77 \\
13. Indecisiveness & 0.42 & 0.71 & 0.60 \\
14. Worthlessness & 0.20 & 0.54 & 0.61 \\
15. Loss of energy & 0.36 & 0.56 & 0.73 \\
16. Changes in sleeping & 0.65 & 0.89 & 0.52 \\
17. Irritability & 0.39 & 0.70 & 0.61 \\
18. Changes in appetite & 0.46 & 0.75 & 0.51 \\
19. Concentration difficulty & 0.40 & 0.69 & 0.69 \\
20. Tiredness or fatigue & 0.50 & 0.57 & 0.51 \\
21. Loss of interest in sex & 0.46 & 0.83 & 0.69 \\
\hline
\end{tabular}

Coefficient alpha 0.93

Mean inter-item correlation coefficient 0.24

$r$ tots- Corrected item-total correlations.

intended purpose and setting of use (17). The Receiver Operating Characteristic (ROC) curve was generated to determine the ideal cut off score for this sample (Figure 1).

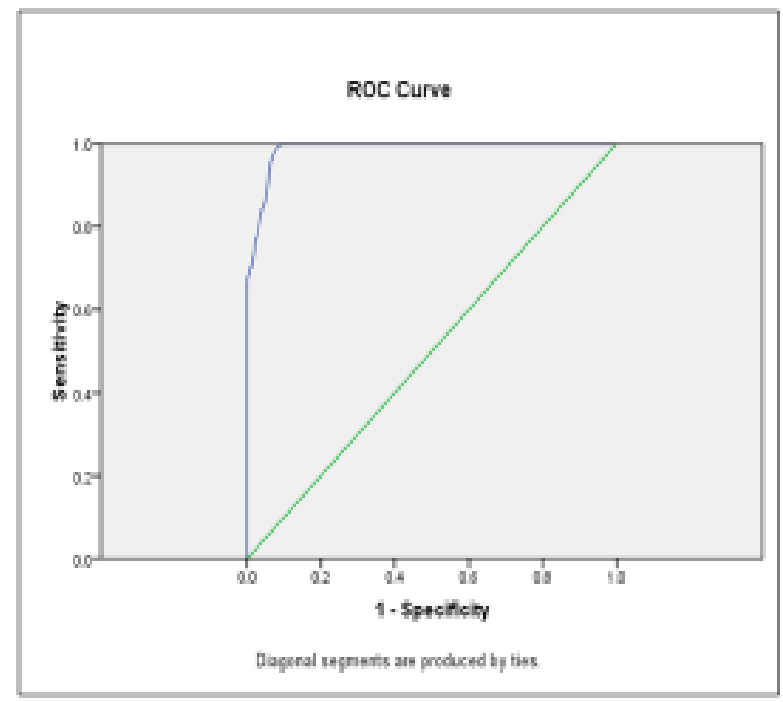

Figure 1. ROC Curve
Table 2. Promax-rotated iterated-principal-factor standardized regression coefficients of the Sinhalese BDI-II Symptom Factor 1 Factor

\begin{tabular}{|l|c|c|}
\hline \multirow{2}{*}{} & \multicolumn{2}{|c|}{ Factors } \\
\cline { 2 - 3 } & $\begin{array}{c}\text { Factor 1 } \\
\text { Cognitive- } \\
\text { affective }\end{array}$ & $\begin{array}{c}\text { Factor } 2 \\
\text { Somatic }\end{array}$ \\
\hline 1. Sadness & 0.61 & 0.44 \\
2. Pessimism & 0.77 & 0.24 \\
3. Past failures & 0.62 & 0.29 \\
4. Loss of pleasure & 0.66 & 0.22 \\
5. Guilty feelings & 0.61 & 0.17 \\
6. Punishment feelings & 0.60 & 0.24 \\
7. Self-dislike & 0.69 & 0.24 \\
8. Self-criticalness & 0.30 & 0.63 \\
9. Suicidal thoughts or & & \\
$\quad$ wishes & 0.59 & 0.39 \\
10. Crying & 0.18 & 0.54 \\
11. Agitation & 0.59 & 0.47 \\
12. Loss of interest & 0.71 & 0.43 \\
13. Indecisiveness & 0.40 & 0.52 \\
14. Worthlessness & 0.75 & 0.26 \\
15. Loss of energy & 0.58 & 0.50 \\
16. Changes in sleeping & & \\
\multicolumn{1}{|c|}{ pattern } & 0.09 & 0.77 \\
17. Irritability & 0.36 & 0.60 \\
18. Changes in appetite & 0.42 & 0.37 \\
19. Concentration difficulty & 0.53 & 0.51 \\
20. Tiredness or fatigue & 0.34 & 0.73 \\
21. Loss of interest in sex & 0.31 & 0.53 \\
\hline
\end{tabular}

A cut-off score of 12 gave a sensitivity of $100 \%$, but was associated with a specificity of $87 \%$. The specificity improved to $91 \%$ at a cut-off score of 14 (Table 3). Based on our findings, a cut off score of 16 appeared to provide the best balance between sensitivity and specificity for the Sinhala translation of BDI-II as a screening instrument. The area under curve for the BDI-II in predicting major depression was 0.98. (95\% CI .98 to .99).

\section{Discussion}

The prevalence of depressive disorder in this study (11.3\%) was much higher than the point prevalence of depression recorded previously (4.6\%) in Sri Lanka (3). The higher prevalence may be due to slightly skewed female: male ratio of the study population and their involvement with community medicine projects of the Faculty of Medicine, Ragama. It was encouraging to note that many participants with depression were currently or previously treated for depression, which is in contrast to previous Sri Lankan studies which have suggested that many people with depression do not seek treatment (6). Higher treatment seeking behaviours in our study 


\begin{tabular}{|c|c|c|c|c|}
\hline Cut off score & $\begin{array}{l}\text { Depressed } \\
(\mathrm{N}=108)\end{array}$ & $\begin{array}{l}\text { Not depressed } \\
(\mathrm{N}=848)\end{array}$ & Sensitivity & Specificity \\
\hline 12 & $216(108)$ & $740(0)$ & 100 & 87 \\
\hline 13 & $189(106)$ & $767(2)$ & 98 & 89 \\
\hline 14 & $180(104)$ & 776 (4) & 96 & 91 \\
\hline 15 & 169 (103) & 787 (5) & 95 & 92 \\
\hline 16 & $150(101)$ & $806(7)$ & 94 & 94 \\
\hline 17 & $145(100)$ & $811(8)$ & 92 & 95 \\
\hline 18 & $131(95)$ & 825 (13) & 88 & 96 \\
\hline
\end{tabular}

(Numbers in parenthesis are the participants who are depressed as determined by gold standard)

population may again be influenced by involvement with the community medicine projects.

Of the individual items, crying had the highest item score followed by sleep, self-criticalness, tiredness and appetite. Worthlessness, suicidal thoughts and selfdislike had the least mean item scores.

The low score on the suicide item may have been influenced by reluctance to reveal suicidal ideation or associated stigma. However it should be noted that 90 participants who were depressed scored 1 or more in this item while only 18 answered in negative. Higher item scores for somatic symptoms of depression were not surprising, as patients with depression in Sri Lanka tend to endorse more somatic symptoms due to cultural and social acceptability $(3,10)$. High scores in fatigability have been observed in similar studies conducted in oriental communities, where it may be considered a more acceptable presentation of depression (20).

The two main religious traditions among the participants of our study are Catholicism and Buddhism. High endorsement of self-punishment reported in similar studies done in Catholic communities may reflect their belief that the suffering associated with depression may be the result of God punishing them for their sins (20). Self-punishment and self-criticalness among Buddhists may be explained by their beliefs regarding past misdeeds causing sufferings as part of karma. Worthlessness and self-dislike, and related constructs were least endorsed. The feeling of inherent self worth on a background of Buddhist teaching may have contributed to this observation.

The item on crying has been highly endorsed in cultures where crying has symbolic value and is appropriate and often expected in certain circumstances (20). Crying is not unacceptable in the Sri Lankan culture. The item on crying is the only item that had no significant difference of mean item scores between depressed and nondepressed participants. Ready endorsement of somatic symptoms and fatigue in depressed patients may lead to diagnosis of physical illness and delay the diagnosis of depression. Hence it is important to have a of high degree of suspicion for depressive disorder and for the use of screening tools in the presence of these complaints.

Our Cronbach's alpha of 0.93 demonstrated that the items of Sinhalese version of the BDI were homogeneous. Studies involving clinical and community samples have consistently confirmed two highly correlated cognitive, affective and somatic factors of the BDI- II $(17,21)$. Initial research by Beck et in a clinical population revealed that cognitive and somatic-affective factors were defined by items 9 and 12 respectively $(17,21)$. In a population study with a sample that consisted of college students, somatic factors were defined by 5 items, and the remaining 16 items loaded on cognitive affective factors (22). The responses of the Sri Lankan adult community sample showed a better fit to the Student Model proposed than to the Outpatient Model. Crying, agitation, selfcriticalness and irritability, which were loaded onto the cognitive affective component in the original study were also loaded on to somatic factor in this study. This may be due to cross cultural variation of manifestation of depression since many with depression present predominantly with somatic complaints in Sri Lanka $(3,10)$.

Cut-off scores were calculated using two main parameters - sensitivity and specificity. When evaluating an instrument for screening purposes, sensitivity should be regarded as more important in order to decrease the risk of false negatives. One negative consequence of screening is that some individuals may test positive for depression on the BDIII, with further evaluation demonstrating that they are not actually depressed. Such false positives result in greater amounts of resources being spent on these individuals. Therefore it is equally important in a country like Sri Lanka with little resources for mental health to minimize over diagnosis of depression. With these considerations in mind, BDI-II total scores of 015 indicated no depression, 16-63 mild to severe depression. A separate cut-off score for mild depression was not calculated as there were only a few participants with mild depression. 


\section{Limitations}

The major limitation of the study is the probable sample bias, since our study population consists of an urban and semi urban population with high health literacy, who were involved with projects organized by the Department of Community Medicine. This may limit the external validity of the Sinhalese BDI II, since the study population may not be representative of patients attending the primary care system of Sri Lanka.

\section{Conclusions}

In conclusion, despite the limitations, this study suggests that the Sinhalese version of BDI II has sound psychometric properties for screening of a Sri Lankan adult population for the detection of depression.

\section{Declaration of interest}

None declared

A Rodrigo, Department of Psychiatry, University of Kelaniya, Ragama

KALA Kuruppuarachchi, Department of Psychiatry, University of Kelaniya, Ragama

A Pathmeswaran, Department of Public Health, University of Kelaniya, Ragama,

Corresponding author: Asiri Rodrigo

Email: asirir2000@yahoo.com

DOI http://dx.doi.org/10.4038/sljpsyc.v6i2.8076

\section{References}

1. Vos T, Flaxman AD, Naghavi M, et al. Years lived with disability (YLDs) for 1160 sequelae of 289 diseases and injuries 1990-2010: a systematic analysis for the Global Burden of Disease Study 2010. Lancet 2012; 380(9859): 2163-96.

2. Bromet E,Andrade LH, Hwang I, et al. Cross-national epidemiology of DSM-IV major depressive episode. BMC Med 2011; 9: 90.

3. Ball HA, Siribaddana SH, Kovas Y, et al. Epidemiology and symptomatology of depression in Sri Lanka: A crosssectional population-based survey in Colombo District. J Affect Disord 2010; 123(1-3): 188-96.

4. Perera B, Torabi MR, Jayawardana G, Pallethanna N. Depressive Symptoms among Adolescents in Sri Lanka: Prevalence and Behavioural Correlates. J Adolesc Health 2006; 39(1): 144-6.

5. Husain F, Anderson M, Lopes Cardozo B, et al. Prevalence of war-related mental health conditions and association with displacement status in postwar Jaffna District, Sri Lanka. JAMA 2011; 306(5): 522-31.
6. Abeyasinghe R, Gunnell D. Psychological autopsy study of suicide in three rural and semi-rural districts of Sri Lanka. Soc Psychiatry Psychiatr Epidemiol 2008; 43(4): 280-5.

7. Samaraweera S, Sumathipala A, Siribaddana S, Sivayogan S, Bhugra D. Completed suicide among Sinhalese in Sri Lanka: a psychological autopsy study. Suicide Life Threat Behav 2008; 38(2): 221-8

8. Saver BG, Van-Nguyen V, Keppel G, Doescher MP. A qualitative study of depression in primary care: missed opportunities for diagnosis and education. J Am Board Fam Med 2007; 20(1): 28-35.

9. Tylee A, Gandhi P. The Importance of Somatic Symptoms in Depression in Primary Care. Prim Care Companion J Clin Psychiatry 2005; 7(4): 167-176.

10. Rajapakse T, Sivapalasingam A. Patients presenting with depression to a psychiatry clinic: a descriptive survey. SL J Psychiatry 2011; 2(1): 36-38

11. Research Triangle Institute. Screening for Depression Systematic Evidence Review. Chappel Hill: University of North Carolina; 2002.

12. Mitchell AJ, Vaze A, Rao S. Clinical diagnosis of depression in primary care: a meta-analysis. Lancet 2009; 374(9690): 609-19.

13. Department of Health. A national service framework for mental health. London: Department of Health; 1999.

14. Baer L, Blais MA. Handbook of clinical rating scales and assessment in psychiatry and mental health. New York: Hanuma Press; 2010.

15. Zimmerman M, Posternak MA, McGlinchey J, Friedman M, Attiullah N, Boerescu D. Validity of a self-report depression symptom scale for identifying remission in depressed outpatients. Compr Psychiatry 2006; 47(3): 85-8.

16. Zimmerman M, McGlinchey JB. Why don't psychiatrists use scales to measure outcome when treating depressed patients? J Clin Psychiatry 2008; 69(12): 1916-9.

17. Beck AT, Steer RA, Brown GK. Manual for the Beck Depression Inventory-II. San Antonio, TX: Psychological Corporation; 1996.

18. Flaherty JA, Gaviria FM, Pathak D, et al. Developing instruments for cross-cultural psychiatric research. J Nerv Ment Dis 1988; 176(5): 257-63.

19. Guillemin F, Bombardier C, Beaton D. Cross-cultural adaptation of health-related quality of life measures: literature review and proposed guidelines. J Clin Epidemiol 1993; 46(12): 1417-32.

20. Wang YP et al. Validation of the Beck Depression Inventory for a Portuguese-speaking Chinese community in Brazil. Braz J Med Biol Res 2005; 38: 399-408.

21. Ward LC. Comparison of factor structure models for the Beck Depression Inventory-II. Psychol Assess 2006; 18(1): 81-8.

22. Whisman MA, Perez JE, Ramel W. Factor structure of the Beck Depression Inventory-Second Edition (BDI-II) in a student sample. J Clin Psychol 2000: 56: 545-55. 